\title{
Effect of silty-sand compressibility on transferred velocity from impulsive blast loading
}

\author{
K. Scherbatiuk ${ }^{1}$, D. Pope $^{2}$, J. Fowler ${ }^{1} \&$ J. Fang ${ }^{3}$ \\ ${ }^{1}$ DRDC Suffield, Canada \\ ${ }^{2}$ DSTL Porton Down, UK \\ ${ }^{3}$ Amtech Aeronautical, Canada
}

\begin{abstract}
A common assumption made in calculating response of walls subjected to blast loading is that the reflected blast loading is often based on rigid body dynamics along the thickness of walls and zero particle velocity at the fluid-structure interface. Of interest to the authors is the development of a quick-running impulse-dominated structural response model for soil-filled concertainer walls that assumes reflected impulse assuming zero particle velocity during the course of loading. The magnitude of response will directly depend on the velocity transferred in the thickness direction by the blast loading. The aim of this study is to assess whether a reduction factor should be applied to reduce the initial velocity corresponding to the zero-particle-velocity reflected impulse for concertainer walls filled with compressible silty-sand. Results obtained from a 1-D numerical program using a simple analytical coupling model are validated with those obtained from a commercial coupled Computation Fluid Dynamics and Computational Solid Mechanics (CFD/CSM) code. Both coupled and uncoupled loading combinations are investigated for rigid body, elastic, and compressible silty-sand material behaviours using the simple 1-D numerical program developed and validated. Graphical results for a range of peak blast pressures are compiled to present the differences in zero-particle-velocity reflected impulse required to attain the same velocity in the thickness direction for each different combination. The resulting differences in charge standoffs between each combination are compared as well for a number of different charge sizes. For this specific problem of calculating transferred velocity in the thickness direction for a silty-sand filled concertainer wall subjected to impulsive blast loading, results that include coupling only lead to a small difference in the resulting charge standoff and therefore reasonable results will be achieved assuming uncoupled loading and rigid body dynamics.
\end{abstract}

Keywords: blast, impulse, soil, sand, rigid-body, compressibility, concertainer, hesco. 


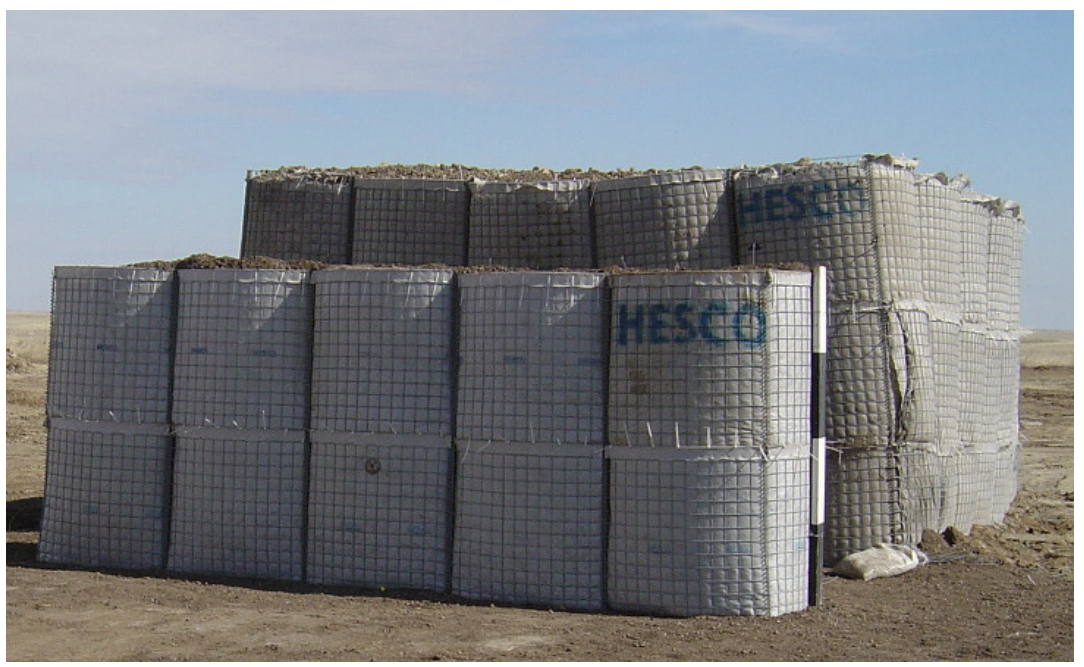

Figure 1: A bunker constructed using soil-filled concertainers.

\section{Introduction}

Defence Research and Development Canada - Suffield is currently undertaking a four year research program which involves the recommendation of expeditionary structures that exhibit improved performance and protection against blast loading. Soil-filled welded-wire-mesh geotextile-lined concertainers are frequently used to construct walls for defensive structures such as the bunker shown in Figure 1. Of interest is the ability to predict the final response of these structures subjected to blast loading.

Accurate results have been achieved by Pope [1] by utilizing a fully coupled commercial CFD/CSM code. Fully coupled calculations involve computing the effect of the loading on the structure and computing the effect of the structures movement on the loading simultaneously. Uncoupled calculations involve computing the loading based solely on the initial position of the structure and then applying the loading to the structure face assuming that the loading does not change regardless of movement at the fluid-structure interface. However computational effort for both coupled and uncoupled calculations can be extensive for physically large structures that are impulse-dominated in their response. Often the size of elements that are used within a computational model for physically large structures cannot be scaled correspondingly larger as well. Thus large numbers of elements are often necessary for physically large structures while constraints in time step size must still be maintained. Impulsively dominated structures also respond very slowly compared to the relative time duration of a typical blast loading, contributing additionally to long computational run times. Hence a need exists for a quick-running concertainer wall response model that yields reasonably accurate results.

Detailed fully coupled fluid-structure models for soil-filled concertainer walls using a commercial coupled CFD/CSM code have been constructed by 
Pope [1] and experimentally validated by Fowler [2]. Shock tube experiments by Murray et al. [3] have been conducted to investigate velocity profiles and shock trajectories attained from a blast wave impacting rigid movable wall. In this study, the accuracy of an analytical solution by Meyer [4] computing the velocity-time history of a rigid movable wall impacted by a shock wave was investigated and found to be in good agreement for weak shock waves, but underestimated reflected loading for strong shock waves. Impulse reduction coefficients have also been investigated by Szuladzinski [5] who studied the effect of varying geometries and mass densities of cross sections on transferred velocity from impulsive blast loading using a commercial coupled CFD/CSM code.

The aim of this paper is to compute, investigate, and compare the magnitude of differences in charge standoffs required for the same velocity in the thickness direction to be attained between uncoupled calculations and coupled calculations for the material types of rigid, elastic, and compressible silty sand. The magnitude of the differences in results will determine if an impulse reduction factor is warranted as a component in the formulation of an analytical/semi-empirical impulse-dominated quick-running blast response model for soil-filled concertainer walls.

To undertake this study, the response a CFD/CSM model of a simple soil-filled concertainer wall is studied in terms of spatially averaged velocity transferred in the thickness direction from an impulsive blast loading. A simple 1-D finite element model is formulated representing a core of soil through the wall thickness using equations from Meyer [4] to couple the blast loading. An equivalent $1-\mathrm{D}$ model is constructed using a commercial coupled CFD/CSM code. The results from the proposed simple 1-D model will be compared and validated with results from the commercial CFD/CSM model in terms of spatially averaged velocity-time histories. An automated root solving procedure that calls the simple 1-D model as a program function will then be derived, coded and developed to solve the inverse problem of calculating zero-particle-velocity reflected pressure and impulse iso-velocity lines for various combinations of uncoupled versus coupled loading, rigid body, elastic, and compressible silty-sand material behaviours. The results plotted graphically on the zero-particle-velocity reflected pressure and impulse plane along with the performance of a range of charge sizes as a function of standoff show the differences in results between uncoupled and coupled loading for the different material behaviours. Magnitudes of differences in standoffs arising from differences in behaviour of the various materials and uncoupled versus coupled combinations will determine whether a reduction factor should be applied to zero-particle-velocity reflected impulses to result in accurate transferred velocities in the thickness direction.

\section{Description of response of concertainer walls}

A model of a simple soil-filled concertainer wall subjected to uniform blast loading was constructed using a commercial CFD/CSM code. Figure 2 compares 
the wall's initial position, its position immediately following the blast loading at a time of $30 \mathrm{~ms}$, and its final position at $700 \mathrm{~ms}$.

The strip outlined in the wall's initial position represents a typical core of soil through the thickness of the wall that is assumed to undergo uniaxial strain during the course of the blast loading. This assumption is made with the exception of the areas of the wall that are too close to any edges to strain uniaxially. Comparison of the initial wall position to its position after the duration of the blast loading indicates that the wall has not attained appreciable deformation at this point. Yet detailed modelling results show that the wall has attained a spatially averaged velocity in the thickness direction of approximately $2 \mathrm{~m} / \mathrm{s}$. The magnitude of deformation of the wall shown in its final position is mostly a result of the momentum imparted to the wall by the blast loading and the subsequent strains and rotations that occur to absorb this momentum. The large differences between the final position and position after the duration of the blast loading indicates that the response of soil-filled concertainer walls is mostly impulse-dominated.

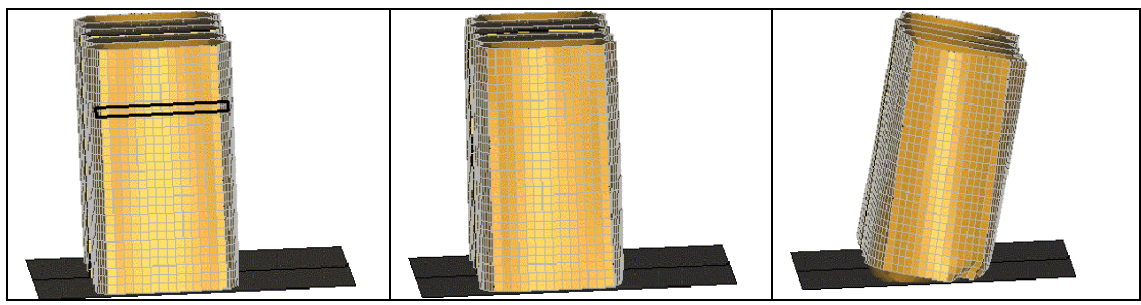

Figure 2: Initial position of wall, position after duration of blast load at $30 \mathrm{~ms}$, and final position at $700 \mathrm{~ms}$.

\section{Validation of a one-dimensional simple coupled model with a detailed coupled CFD/CSM model}

A detailed 1-D shock tube model was constructed using a commercial CFD/CSM code to simulate an incident blast wave impacting a frictionless elastic solid under uniaxial strain and monitor its spatially-averaged velocity-time history. A schematic of this model is shown in Figure 3.

A pressure and temperature of 1 atmosphere and $288 \mathrm{~K}$, respectively, were assumed for the air at ambient conditions while a pressure and temperature of 25 atmospheres and $705.81 \mathrm{~K}$, respectively, were assumed for the air at pressurized conditions. An ideal gas equation of state was used assuming $\gamma$ equal to 1.4. The shock tube extends in both directions for the distances specified to reduce occurrence multiple shock wave reflections, permit the formation of a slight transmitted shock at the opposing end, and to allow for movement of the elastic solid. The density of the elastic solid was assumed to be $1925 \mathrm{~kg} / \mathrm{m}^{3}$. The bulk modulus and Poisson's ratio were assumed as 247.6 MPa and 0.3 , respectively, to amount to a uniaxial confined effective modulus of 400 $\mathrm{MPa}$. 


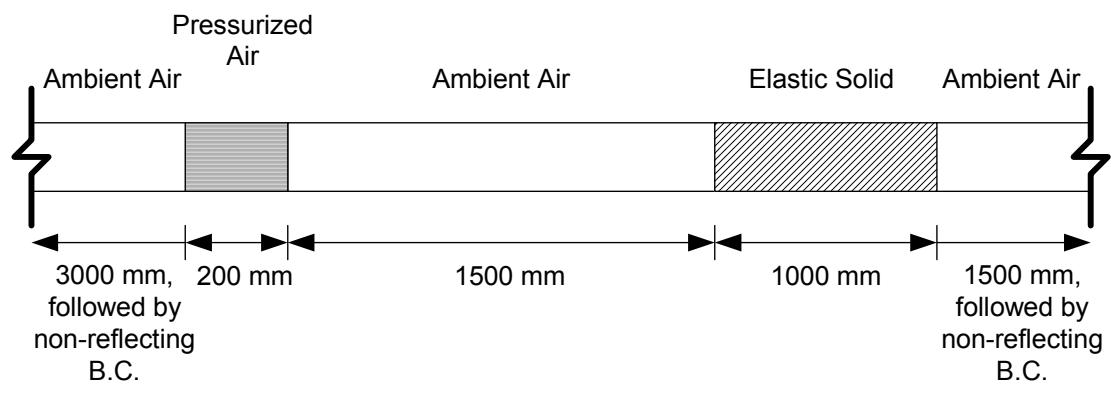

Figure 3: Detailed shock tube model constructed using a commercial $\mathrm{CFD} / \mathrm{CSM}$ code.

A simple 1-D numerical model, shown in Figure 4, was formulated using onedimensional finite elements and equations from Meyer [4] to couple the reflected blast loading.

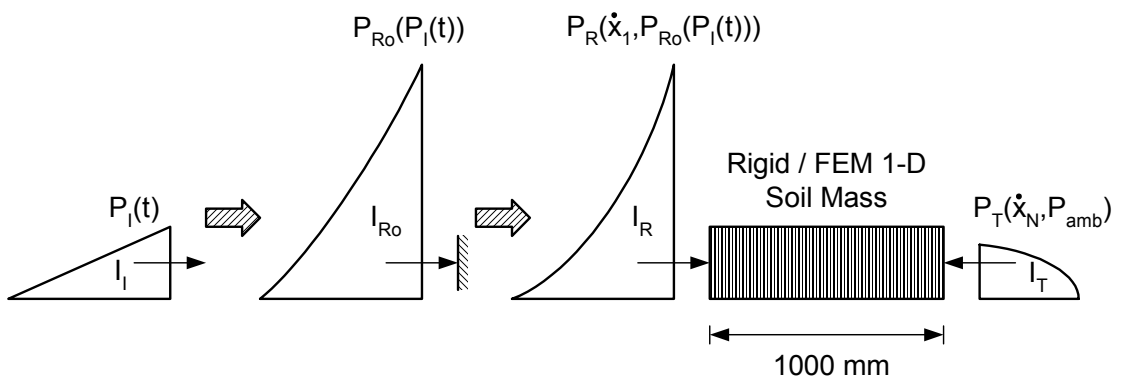

Figure 4: $\quad$ Schematic of simple model.

The parameter $P_{I}$ represents the incident pressure time history while the parameter $P_{R o}$ represents the reflected pressure time history assuming zero particle velocity. The parameter $P_{R}$ represents the actual reflected pressure-time history applied to the fluid-structure interface and is a function of the velocity and temperature at this interface. Coupling on both the shocked side and the opposing side were included into the model. The pressure on the opposing side is represented by $P_{T}$, which is the pressure time history at the right side of the mass, and is a function of the velocity at the fluid-structure interface on the right hand side, and the ambient pressure and temperature. The difference equations were derived, coded, and numerically integrated. The displacement at each time step was solved for using fixed-point iteration. The parameters $I_{I}, I_{R o}, I_{R}$, and $I_{T}$ represent the impulses corresponding to the pressures described. The reflected pressure-time histories and corresponding temperature distributions corresponding to zero-particle-velocity were calculated using the ideal gas equations from Henrych [6]. 
Due to difficulties in mitigating the occurrence of multiple reflections in the detailed CFD/CSM model, the zero-particle-velocity reflected pressure and temperature profile were used as inputs into the simple model instead of the incident pressure and temperature profiles, contrary to what is shown in Figure 4. These profiles were computed using the detailed CFD/CSM model by placing a rigid boundary at the location of the left hand side of the elastic solid. The rigid boundary was removed from the detailed model and elements for the elastic solid and additional fluid elements with ambient air were added on the right had side. Both models contain a hundred elements along the thickness of the elastic solid. The velocity-time histories at each element node of the elastic solid in both models were recorded and spatial averages of these velocities at each increment in time were computed. Figure 5 compares the spatially averaged velocities computed by both models along with the theoretical velocity-time history calculated assuming uncoupled loading and rigid body dynamics.
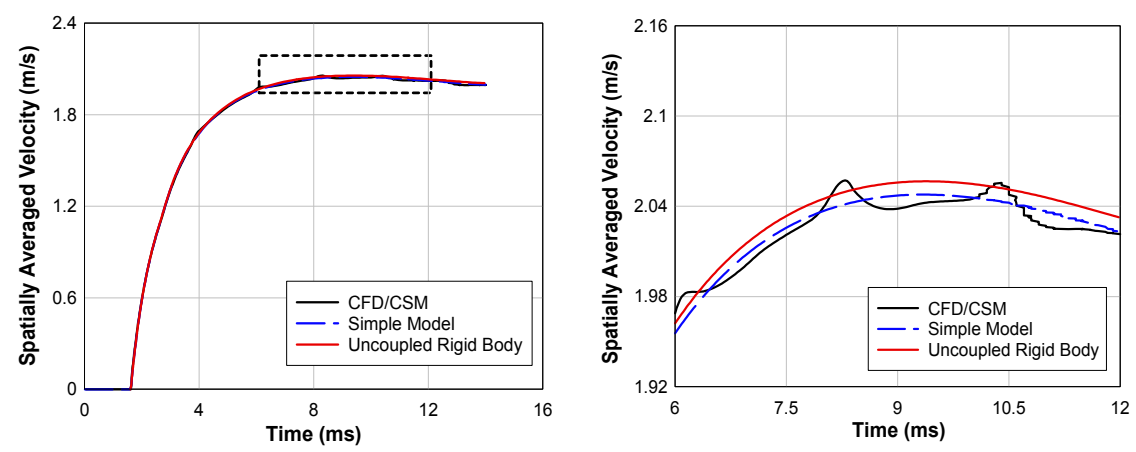

Figure 5: Comparison of spatially averaged velocities for (a) entire profile, and (b) magnification of profile contained within dotted box.

On average, reasonable agreement is achieved between the detailed CFD/CSM model and the simple model, both attaining average velocities short of the velocities calculated assuming uncoupled loading and rigid body dynamics. The author is uncertain why the spatially averaged velocities computed using the detailed CFD/CSM model appear to oscillate, although the timing of the oscillations do appear to correspond with the time it takes for a compressive wave to travel from one side to the other.

The simple model was created to expedite the computing of velocity results, and to provide well-defined distributions of pressure waves of a specified incident triangular shape, zero time to rise, and absence of a negative phase so that compounded effects from variables not intended to be examined in this study could be eliminated. Although some of these real effects may be valuable to examine, the complexity in the presentation of the results are greatly increased with even one added dimension created by an additional variable. 


\section{Analysis and discussion of differences in coupled and uncoupled results}

In developing a quick-running, impulse-dominated blast response model, it is important to quantify what differences exist in transferred velocities between the cases of uncoupled versus coupled loading and their corresponding effects on charge standoffs. The following analysis is aimed to quantify the differences in charge standoffs required to produce identical velocity results if one assumes a rigid material along the thickness, versus an elastic material or compressible silty-sand. Thus the six different cases that are compared are uncoupled-rigid, uncoupled-elastic, uncoupled-compressible, coupled-rigid, coupled-elastic, and coupled-compressible.

The uniaxial stress-strain relationship assumed for a compressible silty-sand is shown in Figure 6. This uniaxial stress-strain relationship and the material coefficients were approximated based on engineering judgement of actual uniaxial compression test results within the MPQW soils database [7]. The effective uniaxial moduli $E_{1}$ and $E_{2}$ were selected as $0.4 \mathrm{GPa}$ and $100 \mathrm{GPa}$ respectively. The limiting strain $\varepsilon_{a}$ was selected as $25 \%$. For tensile strains, a uniaxial effective modulus equal to $E_{1}$ was selected and the maximum cohesive tensile stress limit was selected as $50 \mathrm{kPa}$. The density of the soil for all models as well as the rigid body calculations was selected as $1925 \mathrm{~kg} / \mathrm{m}^{3}$. For calculations involving an elastic solid material type, effective uniaxial modulus $E_{1}$ was assumed. The thickness of the soil mass was assumed as $1 \mathrm{~m}$ for the entire analysis.

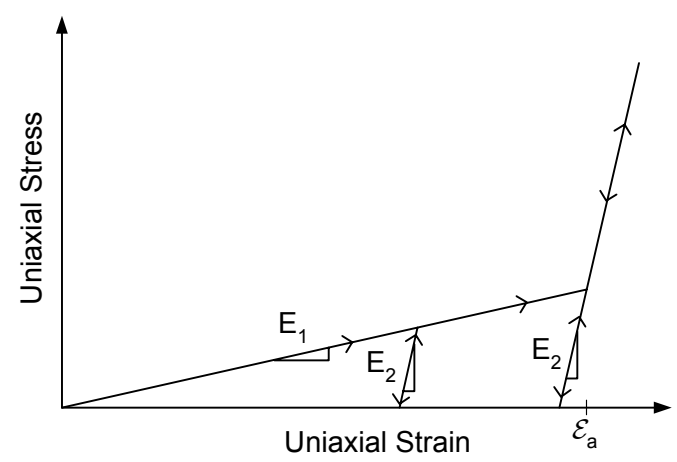

Figure 6: Uniaxial stress-strain relationship for silty-sand.

There are a number of possible ways one may study the differences in transferred velocity between the six cases. The easiest way would be to use the same incident loading profile and compare differences in transferred velocities for each case. However it is likely that a quick-running model would utilize zero-particle-velocity peak reflected pressure and positive impulse calculated from simple blast loading reflection models as an input. This warrants a 
comparison of the differences in the zero-particle-velocity reflected impulse necessary to accelerate all six cases to equal velocities in the thickness direction. This is more computationally demanding and requires a root solving scheme to find the solution to this inverse problem. But the variation of results can be easily and directly compared to charge performance with respect to variations in standoffs.

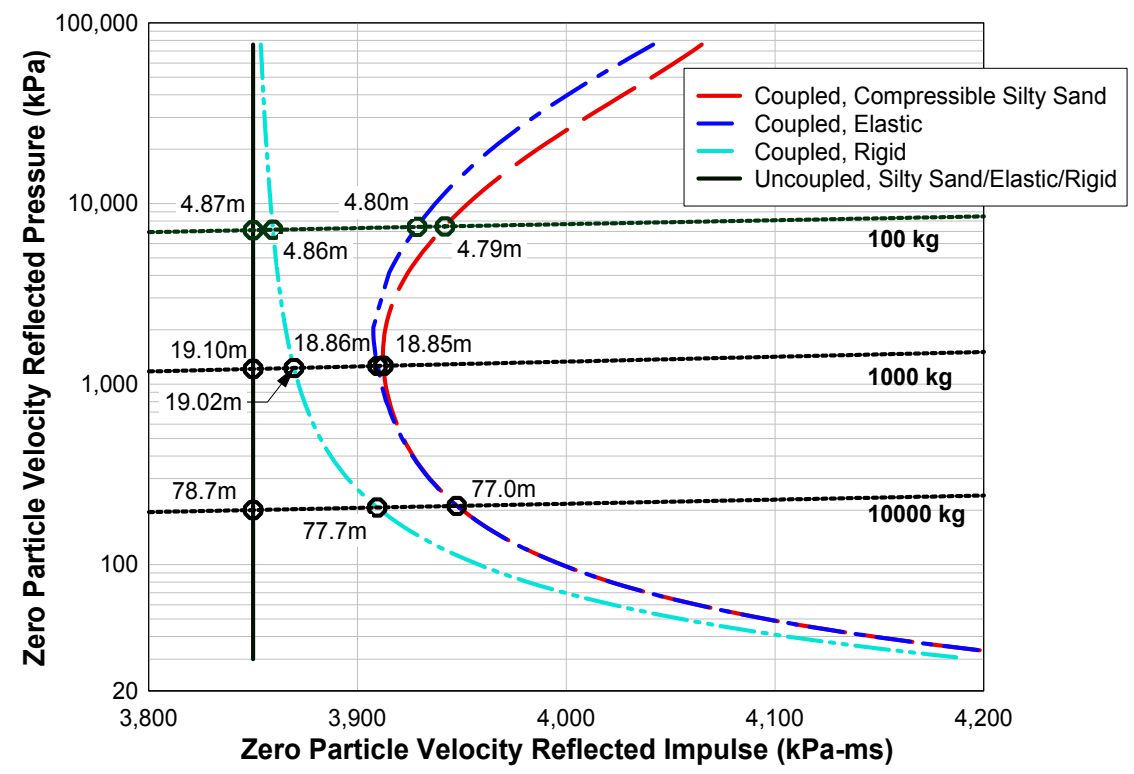

Figure 7: Zero-particle-velocity reflected impulse and pressures required to accelerate a $1 \mathrm{~m}$ thick mass to a velocity of $2 \mathrm{~m} / \mathrm{s}$ in the six different calculation cases.

Figure 7 shows the results specified in required zero-particle-velocity peak reflected pressure and impulse to accelerate the six different cases to a velocity of $2 \mathrm{~m} / \mathrm{s}$. The simple 1-D numerical model described in the previous section was utilized along with an automated root-solving procedure to compute these results. The coupling was turned off in the numerical program to compute the results involving uncoupled calculations. Calculations were carried out assuming a triangular shaped incident blast wave up to the duration time of the blast loading and the spatially averaged velocity was noted at this point. If the peak incident pressure and incident positive impulse selected by the root solver yielded a spatially-averaged velocity of $2 \mathrm{~m} / \mathrm{s}$ within the tolerances of the root solver, the zero-particle-velocity reflected pressure and impulse were calculated for the corresponding incident blast loading and were plotted on the graph to generate the curves shown. Parametric curves in standoff for zero-particle-velocity reflected impulse and pressure for charge sizes of $100 \mathrm{~kg}$, 
$1000 \mathrm{~kg}$, and $10000 \mathrm{~kg}$ of TNT in a hemispherical configuration were produced using Blast Effects Computer version 5 [8] and imposed onto the graph.

Note that the magnitude of differences between the lines representing the different cases appear visually exaggerated due to the choice of scale range on the ordinate axis. Also for peak reflected pressures above $5000 \mathrm{kPa}$, the ideal gas laws and assumptions made in Meyer [4] used to compute the coupling begin to lose validity. The true magnitudes of differences in the lines at higher peak pressures may be somewhat larger but in absence of a more robust model, the author felt it was important to include these ranges of computations to show the general shape of the curves and how they deviate from one another.

All three uncoupled cases of rigid, elastic, and compressible generated identical results. Thus it can be concluded that, for an uncoupled calculation, since the loading is unaffected by the movement at the fluid-structure interface, regardless of the amount of internal deformation that occurs along the thickness, a specified reflected impulse will always yield the same spatially-averaged velocity. The additional strain energy taken up by the internal deformation is balanced with the additional applied work of the loading moving through the range of motion at the fluid-structure interface. These results agree with the equation $v=I_{R o} / m$, where $v$ is the average velocity in the thickness direction, $I_{R o}$ is the zero-particle-velocity reflected impulse, and $m$ is the mass of the thickness of material. The numerical program was checked to see that this output was reached when the coupling was turned off and a rigid mass was used instead of a compressible one. The results were in perfect agreement within the numerical tolerances of the root solver.

At very high peak pressures, the coupled-rigid line approaches the uncoupled-rigid line. This is because the higher the particle velocity of the blast wave, the less significance the comparatively low particle velocity at the fluidstructure interface affects the relief of the loading. For lower reflected pressures with correspondingly lower particle velocities, the particle velocity at the fluid-structure interface becomes comparatively more significant and has increased effect. Observing the lower right-hand-side portion of the coupled-rigid, coupled-elastic, and coupled-compressible lines, these lines will eventually approach a horizontal asymptote. As impulse increases to infinity, these loadings approach ideal step shock waves. In theory, an abject that is hit by an ideal step shock wave of infinite impulse will not approach infinite velocity, but approach a maximum velocity limited to the particle velocity of the incident step shock wave.

On the lower right-hand-side of the graph, the coupled-elastic and coupled-compressible lines approach the coupled-rigid line because the pressures are not of a high enough magnitude to cause much internal deformation. Therefore the behaviour of the coupled-elastic and coupled-compressible lines approach the coupled-rigid line for larger charges at further standoffs. For higher pressure, short duration loadings resulting from smaller charge sizes at closer standoffs, the contribution of rigid body velocity becomes very small while the contribution of material deformation increases. Internal deformation is much larger, which causes more movement at the fluid-structure interface and more 
relief of the loading. Thus more deviation between the coupled-elastic and coupled-compressible lines is seen when compared to the coupled-rigid line. A full discussion of energy losses is beyond the scope of this paper, but the source of these differences more come from relief to the blast loading rather than energy lost due to strain energy absorption. Since all of the uncoupled results regardless of internal deformation received the same applied impulse at the fluid-structure interface, than it follows that in the coupled calculations, although the incident loadings and corresponding zero-particle velocity reflected impulses may be different in all cases, the fluid-structure interface in all cases received an identical amount of applied impulse. Tracking the actual applied impulse at the interface in the coupled calculations validated this. It was the material deformation that caused differences in the actual applied loading.

The differences in standoffs produced by this coupling effect are demonstrated by calculating at what standoffs lines of charge performance intersect the equal-velocity lines for the six cases. For this specific problem, the highest difference produced by this effect was the discrepancy between the $78.7 \mathrm{~m}$ standoff and the $77.0 \mathrm{~m}$ standoff for the $10000 \mathrm{~kg}$ charge, which amounts to a negative $2.16 \%$ difference. To summarize, the magnitude of differences in standoffs between uncoupled and coupled calculations for this specific problem of transferred velocity to soil-filled concertainer walls are not large. Therefore the need to account for this behaviour in a quick-running model is small and reasonable results will be achieved if an initial velocity in the thickness direction is calculated using the zero-particle velocity reflected impulse and ignoring the effects of coupling.

\section{Conclusions}

The aim of this study was to assess whether reduction in the transferred velocity corresponding to the zero-particle-velocity reflected impulse is necessary for concertainer walls filled with compressible silty-sand. A quick-running 1-D model using equations in Meyer [4] to couple the blast loading was formulated. The results were compared to results from a fully coupled commercial CFD/CSM code and appear to be in reasonable agreement. The six cases of uncoupled-rigid, uncoupled-elastic, uncoupled-compressible, coupled-rigid, coupled-elastic, and coupled-compressible were investigated. Regardless of the amount of material deformation in the thickness direction, an uncoupled calculation will always yield the same velocity in the thickness direction. For this specific problem of transferred velocity in the thickness direction to a soil-filled concertainer wall, coupling does make a clear difference in results. However the magnitude of this effect only contributes to less than a few percent difference in the charge standoff, and therefore is not worth considering in the formulation of a quick-running impulsive dominated model. Using the zero-particle velocity reflected impulse to calculate the initial velocity in the thickness direction is a reasonable approximation. 


\section{References}

[1] Pope D. J. The Use of Explicit Element Analysis to Simulate the Behaviour of Military Structures Exposed to Far-field and Near-field Blast Loading, Technical Report, QinetiQ, UK, pp. 1-52, 2004.

[2] Fowler J. Personal communication, 15 June 2005, Defence Scientist, DRDC Suffield, Medicine Hat, Alberta, Canada.

[3] Murray, S. B., Zhang, F., Gerrard, K. B., Guillo, P., and Ripley, R., Influence of Diaphragm Properties on Shock Wave Transmission, Proceedings of $24^{\text {th }}$ International Symposium on Shock Waves, Beijing, China, July 11-16, 2004.

[4] Meyer, R. F., The Impact of a Shock Wave on a Movable Wall, Journal of Fluid Mechanics, 3, pp. 309-323, 1958.

[5] Szuladzinski G. Personal communication, 18 March 2005, Principal, Analytical Service Party Ltd., Sydney, Australia.

[6] Henrych, J., The Dynamics of Explosions and Its Use. Elsevier: New York, pp. 159-163, 1979.

[7] $M P Q W$ v1.0. U.S. Army Engineer Waterways Experiment Station, Structural Mechanics Division, Vicksburg, US, 1995.

[8] Swisdak, M., Ward, J., DDESB Blast Effects Computer BEC v5.0. Explosives Safety Board, Department of Defence, 2001. 